$\mathbb{P}$ periodica polytechnica

Electrical Engineering

$55 / 1-2$ (2011) $71+80$

doi: 10.3311/pp.ee.2011-1-2.08

web: http://www.pp.bme.hu/ee

(c) Periodica Polytechnica 2011

RESEARCH ARTICLE

\section{General mobility modeling and location prediction based on markovian approach constructor framework}

\author{
Tamás Szálka / Péter Fülöp / Sándor Imre
}

Received 2012-02-13

\begin{abstract}
Nowadays, in the wireless networks the number of users and the transferred packet switched data are increasing dramatically. Due to the demands and the market competition the services are becoming more complex, therefore network providers and operators are facing even more difficult network management and operation tasks. The efficient network dimensioning and configuration highly depend on the underlying mathematical model of user distribution and expected data transfer level. In this paper we propose a Markov Movement-model Creator Framework (MMCF) for setting up a model based on the network parameters and requirements with optimal number of states. Firstly we describe a method that gives an abstract model of the mobile network and the node, and we introduce a simple classifying method that defines the necessary parameters of the exact Markov movement model. The mathematical solutions for determining these parameters are also presented in the paper. Finally we analyze the accuracy, complexity and usability of the proposed MMCF and an analytical comparison is made with other mobility models, the comparison is justified with simulations. The movement model created with the framework helps the network operators in setting up an effective authorization, fraud detection system or solving self-configurations issues.
\end{abstract}

\section{Keywords}

Wireless network $\cdot$ mobility $\cdot$ Markov model $\cdot$ location prediction

Tamás Szálka

Péter Fülöp

Sándor Imre

Department of Telecommunications, BME, H-1117 Budapest, Magyar tudósok krt. 2., Hungary

\section{Introduction}

Information mobility has become one of the most common services in the modern world with the widespread usage of portable phones and other smart, mobile devices. The number of users and the amount of the transferred data is increasing dynamically and there are more and more technologies, standards, and solutions to support mobility. The providers offer different and sophisticated services in current market competition. Hence the network providers and operators face more and more complex management systems and operation tasks. Wireless multimedia and other services have many requirements and the resources in the network are often expensive and limited. Nowadays the operation tasks have some critical parts, i.e. guaranteeing the security of user-related information and data, providing QoS and maintaining the service levels in the network. Many solutions based on movement mobility models are provided in the literature for these tasks. The enhanced radio techniques applied in mobile systems provide enough bandwidth for present mobile multimedia applications. However such applications are sensitive to the degradation of QoS parameters. To avoid such degradation two important issues should be considered; proper dimensioning of physical network resources is essential and by using Call Admission Control (CAC) the operator can limit the number of newly accepted connections, thus achieve traffic shaping [1]. Accurate, yet simple mobility models are required to address these issues. In recent years people increasingly rely on wireless devices in their daily life for very sensitive tasks such as shopping and bank transactions. Although many authentication protocols are used in wireless, mobile networks, it is still a challenging issue to design a fully secure mobile environment because of the open radio transmission environment and the vulnerability of mobile devices. Anomalybased detection as part of the detection-based techniques creates normal profiles of system states or user behaviors, stores and periodically compares them with the current activities. If significant deviation is detected, the network system raises an alarm. However a user profile is very difficult to build up, it could largely increase the security of a wireless system [2]-4]. Mobility models and location prediction could take significant 
parts of creating user profiles as well.

The previously mentioned fast evolution of network applications and services require skilled individuals to install, configure and maintain these systems. Other possibility is to introduce mechanisms and procedures, which make a system to be able to reconfigure, heal or install itself [5]. These systems shall be capable of modifying their own behavior and adapt to environmental changes based on performance measures. However this area is not well explored, a well developed mobility model can be used as a proper trigger to rebuild a cell boundary in $4 \mathrm{G}$ LTE (Long Term Evolution) network or recognize radio interface problem of mobile access points.

Different areas have been introduced above where mobility models should be used, but the scope of this research area is far more wider nowadays. The discussion of individual or group mobility modeling problems is a hot topic these days [4, 7, 8, 10, 14, 16]. Beside other approaches a few propositions are using Markov model as a sophisticated mathematical solution [8, 10, 13, 14]. In this paper our aim is to give general design guidelines to create a Markov movement mobility model with optimal number of states and proper accuracy according to the network and user movement parameters. To achieve this goal we analyze and classify the Markov approaches from the literature in Chapter 2. We determine common parameters and propose a method to determine and calculate parameters from given network topologies in Chapter 3. These parameters are applied in our algorithms to determine some basic attributes that define a specific Markov movement model. In Chapter 4 we propose a new classification method for Markov approaches in the literature. In Chapter 5 we introduce the Markov Movement-model Creator Framework (MMCF), which is a general algorithm to create an optimal model for an arbitrary network. In Chapter 6 we show a simulation environment that is able to compare different mobility models. Chapter 7 consists of our conclusions.

\section{Related work}

Different mobility models have been proposed in the literature to cope with user mobility in different wireless and mobile networks (e.g. cellular networks, ad hoc networks etc.). In this chapter we give a short overview about mobility models.

In the traditional Random Walk mobility model the node moves from its current location to a new location by randomly choosing a direction and a speed. The Random Walk model defines user movement from one position to the next with randomly selected speed and direction. Many derivatives of the Random Walk mobility model have been developed including one, two, three - and d-dimensional walks.

A flexible mobility framework for hybrid motion patterns is the Mobility Vector model [7]. A mobility vector expresses the mobility of a node as the sum of two subvectors: the Base Vector $(\mathrm{BV}) \mathbf{B}=\left(b x_{v}, b y_{v}\right)$ and the Deviation Vector (DV) $\mathbf{V}=\left(v x_{v}, v y_{v}\right)$. The BV defines the major direction and velocity of the node while the DV stores the mobility deviation from the base vector. The mobility vector $\mathbf{M}$ is expressed as $\mathbf{M}=\mathbf{B}+\alpha \mathbf{V}$ where $\alpha$ is an acceleration factor.

The location history traversed by a mobile user is exploited in High-Order Markov Model that is described in [4,8]. The model focuses on the identification of a group of especially harmful internal attackers. The order- $o$ Markov predictor assumes that the location can be predicted from the current context, which is the sequence of the previous $o$ most recent characters in the location history.

F. Lassabe et al. present a mobility model adapted to the logging of mobile positioning or to the tracking of mobiles. This model is based on the All-Kth Markov Model [10]. They present two predictive models from the AKMM: the K-to- 1 past Model and its improvement, the $K$-to- 1 past* Model. The model defines a Markov state-space constructed of the possible user trajectories. Each state describes a trajectory section of 1 to $\mathrm{K}$ previous locations. The model predicts future locations based on the possibilities of each transition between states. A threshold value is used to select a group of locations which are likely to be visited in the next step, so a handoff procedure can be prepared for each one.

Shiang-Chun Liou et al. present a mobility model with twotier cell structure in [15]. The user trajectory is defined based on the logical function of velocity, direction, acceleration and position. This logical function is converted to a model that uses three preceding geographical locations to estimate the fourth parameter. The location prediction with this estimation enables the network operator to make preparations for a future handoff in the group of cells that are likely to be crossed. Two-tier cell structure is used to decrease the waste of bandwidth due to reserved resources of a future handoff. The two tiers can be described in a mobile cell as a function of distance from the base station (first tier). While the mobile node is close to the base station, it is unlikely that the even with a sudden trajectory modification the mobile node steps into an other cell. On the other hand, if the mobile node is more close to the cell boundary (second tier), the possibility of a handoff is increasing.

Sándor Szabó proposes a ring-based mobility prediction and resource reservation algorithm in [14]. A cell cluster is divided into three cell groups, where the first group is equivalent to the central cell of the cluster, the second and third groups consist of the cells that are located in the first and second cell ring around the central cell respectively. The pre-handoff resource reservation is derived from the possibilities of the event that the mobile node steps from the central cell into a cell of the second or third cell-group. This approach can be considered as the generalization of the two-tier cell structure described in [15] to an inter-cell level.

W. Ma et al. propose a user mobility pattern (UMP) based model (Mobility Patern-Based Scheme - MPBS) in [16]. The MPBS is a general method to follow users in the network without expensive paging operations if the user meets some requirements. The model defines a personal mobility pattern list which 
consists of a sequence of register areas (RA, i.e. mobile cells), and a time-sequence of the trajectory on the RA sequence. The time-sequence is built up by the timestamps of handoffs between RAs, and the dwell times for each RA. Based on the time- and RA-sequence an exact timeline can be defined which is followed by the user. The operator does not need to page the user in different RAs, because the timeline shows that in which RA is the user located at the actual timestamp. Naturally, the ideal user who always follows the timeline does not exist, but the timesequence and RA-sequence provides information even if the actual timeline differs from the prerecorded one. A categorization is presented with four categories where the first category is the ideal user with a timeline-compatible trajectory. Second category involves users who are following the RA-sequence but with time delays or hurries, that is the network operator can find the user in the remaining RA set after the last paging or location update. Users who are located in the appropriate RA set, but are not following the sequence are in the third category. The fourth category is for the users who are located out of their UMPs, that is their actual trajectories are not close to the prerecorded ones.

\section{Network abstraction and determining parameters}

In this Chapter we collect the most significant properties and parameters of the mobile network that can describe an abstract network model.

\subsection{Basic notations and descriptions}

We define the basic notations that we use is the article. The basic model will resemble to the abstract one in our previous work [11, 12].

- The specific network with all its parameters is denoted with N.

- The Mobile Nodes (MN, alias mobiles, moving entities, users) are the mobile equipments that want to communicate with other mobile nodes or fixed partners and move between the radio access points. The number of users (number of MNs) in the model is denoted by $n_{u}$.

- There are Mobility Access Points (MAP, alias cells), these are the only entities that are capable to communicate with the mobile nodes via radio interface. All mobility access points have their own geographical area. While the MN moves in an area, it is always connected to the owner of the area. The number of mobility access points in the model is denoted by $n_{m}$. The user can connect to MAPs with handovers from the neighboring MAPs, each user is connected to only one MAP at a time. The neighbor MAPs could use even different access technology than the current MAP and they could be located at the very same geographical place as well, the model does not require one access technology in the whole network. The number of neighbor MAPs of $\mathrm{MAP}_{i}$ is denoted by $n_{n m}^{i}$.
- There are other network elements which provide the communication in the core network behind the MAPs. We denote these as Network Elements (NE).

- Network trace is abstraction of the network operation log, it contains 4-tuples of a timestamp, user ID, MAP ID and network event. A trace entry could mean for example the selected user connected with handover to the MAP at the given timestamp. The network trace contains all information of the mobility of the users in the network.

(Where 'n' is used as notation, it always denotes number.)

\subsection{Deriving parameters of a given network}

A way to describe a network is to observe the network trace. We introduce a method to process the network traces to calculate typical parameters of the mobility.

The trace entry describes the events in a cellular network. An event might be a status change of the given user (e.g. mobile node is idle state, voice call or data transfer is set up, cell boundary crossing). The logical location of the event is determined with the MAP ID where the user is located at the timestamp of the event. (Table 1). The events are recorded in the network management system's logs, thus the information can be extracted from the management system of cellular mobile networks.

The aim is to derive the parameters of the user mobility, therefore we should pick the relevant entries from the network trace. In our work we focused on location changes of users, handovers, and initializing or receiving calls. These events are observed during a time interval that is considered to be the reference interval for deriving model parameters.

We assumed that the user distribution in the network is given at the first moment of the reference interval. We created a discrete sample series where samples are taken with $\Delta \mathrm{t}$ time, that is a location status is assigned to every users per $\Delta \mathrm{t}$ time. $\Delta \mathrm{t}$ is defined system-wide as the minimum of the time interval elapsed between two events registered to the same user. That is the sample frequency is set to the "fastest" user in the network. This ensures that every user event and all status reports are processed.

With this sampling a MAP ID and a status can be determined to every user in every timeslot. The sampling results an $n_{u} \times n_{T}$ sized $P$ matrix, where $n_{T}$ denotes the number of timeslots, and $n_{u}$ the number of users in the model. $P$ matrix stores the MAP IDs and status of each user in each timeslot.

The relative frequency of any status can be determined based on $P$ matrix, for instance the relative frequency of receiving voice call in a MAP, or even the handover rate between two different MAPs. We defined the $S$ set, which contains all possible states and events appearing in the logs. The important ones are the following:

- receiving voice call

- receiving data call 
Tab. 1. An example of wireless network management system's log

\begin{tabular}{llll}
\hline TIMESTAMP & USER ID & MAP/CELL ID & STATUS (OR EVENT) \\
\hline 09:21:43:12 & 41 & 4951 & Idle \\
09:21:45:48 & 41 & 4957 & Idle \\
09:21:49:21 & 41 & 4957 & Voice call \\
09:21:50:38 & 19 & 5341 & Data Call/ Traffic class 2 \\
09:21:51:42 & 84 & 7120 & Idle \\
09:21:51:58 & 19 & 5348 & Data Call/ Traffic class 2 \\
09:21:52:26 & 19 & 5348 & Idle \\
\hline
\end{tabular}

- initiating voice call

- initiating data call

- fall back into idle status.

Depending on the detail of the logs more and different statuses, events could be investigated as well. For example if more data traffic classes are identified in the logs, then they could also be differentiated. In our presented work the statuses mentioned above are sufficient in the model.

To determine the relative frequencies of statuses in a MAP, the status must occur frequently enough, otherwise it is neglected. Let us define the $n_{u} \times n_{C} C^{s}=\left[c_{i, j}^{s}\right]$ matrix, where $c_{i, j}^{s}, s \in S$ is the occurrence of $s$ status with user $i$, in MAP $j$ from the $P$ matrix. The average occurrence of $s$ status in the network is:

$$
c^{s}=\frac{\sum_{\forall i} \sum_{\forall j} c_{i, j}}{n_{u} \cdot n_{m}}
$$

The parameter $c^{s}$ can be used as a main requirement in order to create valid model based on the network. So the network must be monitored for sufficient time before we create a model from it.

Parameter $\varepsilon_{c}$ denotes the minimal occurrence of a status for acceptance, if the occurrence is smaller than $\varepsilon_{c}$, the rate will be 0 . Based on this, the relative frequency matrix of a status can be determined as the following:

$$
\begin{aligned}
& D^{s}=\left[d_{i, j}^{s}\right]=\left\{\begin{array}{ll}
0, & \text { if } c_{i, j}^{s}<\varepsilon_{c} \\
\frac{c_{i, j}^{s}}{c_{j}^{s}} & \text { otherwise }
\end{array} ;\right. \\
& c_{j}^{s}=\sum_{\forall i} c_{i, j}^{s}, s \in S
\end{aligned}
$$

In fact $d_{i, j}^{s}$ denotes the probability the status $s$ occurs when user $i$ is in MAP $j$.

The rate of receiving call is introduced: $\mu$. It can be determined for every user in every MAP from the $D^{\mu}=\left[\mu_{i, j}\right]$, but the average value is also calculable in similar manner described above:

$$
\mu=\frac{\sum_{\forall i} \sum_{\forall j} \mu_{i, j}}{n_{u} \cdot n_{m}}
$$

The most important event is the handover. Let us assume that the aggregated behavior of the MNs can be modeled with a finite state continuous Markov chain. The chain is given with a rate matrix $B_{Q}=\left[b_{i, j}\right]$. In this matrix, all the possible MAPs are listed, which the users earlier have connected to. The matrix will be of size $n_{m} \times n_{m}$, where each $b_{i, j}$ denotes how freque the user steps from $\mathrm{MAP}_{i}$ to $\mathrm{MAP}_{j}$. From the rate matrix the transition matrix $B_{\Pi}$ can be determined easily. We assume that the matrix $B_{\Pi}$ is irreducible and aperiodic that implies that the chain is stable and there exists a stationary distribution. This will be denoted by a density vector $b$. In this vector, the $i^{\text {th }}$ element denotes the probability of the MN or MNs being located under the $i^{\text {th }}$ MAP. This is the same probability as the relative number of handovers from and to the $i^{\text {th }}$ MAP.

If we sum up the $i^{\text {th }}$ row in this matrix, we get a rate of that the MN moves from the $i^{\text {th }}$ MAP. Let $\lambda$ denote the average rate of handover and denote the rate of handovers for a general MN anywhere in the network. The $B$ matrices can be determined for a single user, user group or all of the users as well and it can be assigned to a state in the network also. According these assumptions, for example $B_{\Pi}^{i, s}$ is the transition rate matrix of user $i$, when it is in status $s$.

Another network describing parameter, which is useful during the modeling, is the number of visited MAPs by the user. That is calculated as follows:

$$
n_{v m}=1 \cdot \operatorname{sign}\left(B_{\Pi} \cdot 1\right)
$$

A general network describing parameter, the weighted average of visited MAPs is:

$$
w_{v m}=\frac{\sum_{\forall i} b \cdot \operatorname{sign}\left(B_{\Pi}^{i} \cdot 1\right.}{n_{u}}
$$

The structure of the mobility model will be built up using two main parameters. One is the number of nodes: $n_{c}$. The other one is the average number of neighbor MAPs that should be also weighted with the probability density of the MN:

$$
w_{v n m}=\frac{b \cdot \operatorname{sign}\left(B_{\Pi}\right) \cdot 1}{n_{u}}
$$

When talking about an existing network, the parameters described in this chapter can be calculated easily, producing the base of the model.

\section{Classifying Markov mobility models}

\subsection{Basic assumptions}

In a simple, general Markov-chain based mobility model a user or users can be located in different Markov states. MAP or 
group of MAPs (or merged MAPs based on a special relationship) is mapped to a state or more states of the Markov-chain model. Let us define $X(t)$ random variable, which represents the movement state of a mobile terminal during timeslot $t$. The transition probabilities of the Markov model can be determined from the describing parameters of the network (see Chapter 3 ). Let us assume that the Markov chain is always irreducible and aperiodic, so the stationary user distribution is determinable.

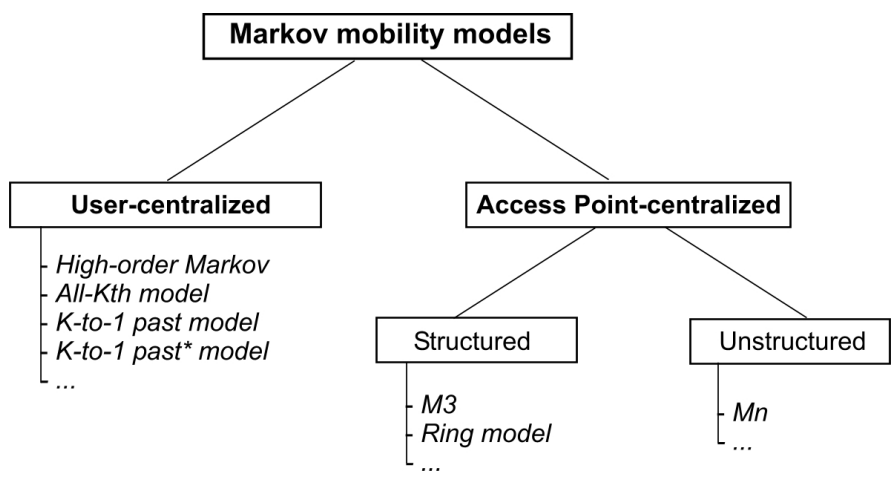

Fig. 1. The main classifying of the Markov mobility models

Two main type of Markov mobility models are distinguished, User-Centralized and Access Point-Centralized. The latter one is further separated into two subtypes. Figure 1 depicts this main classification.

In the next subsection these groups of models are explained with some examples.

\subsection{User-centralized Markov models (UC)}

A user or a group of users from the network is selected for observation in user-centralized Markov models. The users' movement behavior is modeled with a Markov model. Only the MAPs which are visited by the selected user(s) are taken into account, other MAPs, and other users do not affect the structure of the movement model.

Fig. 2 a shows how to create a user centralized model. The chosen user in the example visits only the MAPs between ID 1 and ID 5. Each MAP is mapped into one standalone Markov state. This is a very simple model, where the stationary distribution of the Markov chain is equal to density vector $b$ which is the stationary distribution of $B_{\Pi}$ transition matrix.

The usage of this model is reasonable if the behavior of the user is to be investigated or a user profile is needed to be created for example for fraud detection.

Most of the Markov mobility models in the literature can be classified into this section, for example [4, 9, 10$]$.

\subsection{Access Point-centralized Markov models (AC)}

The access point-centralized Markov models can be used when the user distribution in a selected MAP or group of MAPs must be determined. Instead of modeling the behavior of an individual user, a MAP and its environment is to be observed. In these cases a MAP or more MAPs and defined neighbors are selected according to a requirement. The users who stepped into
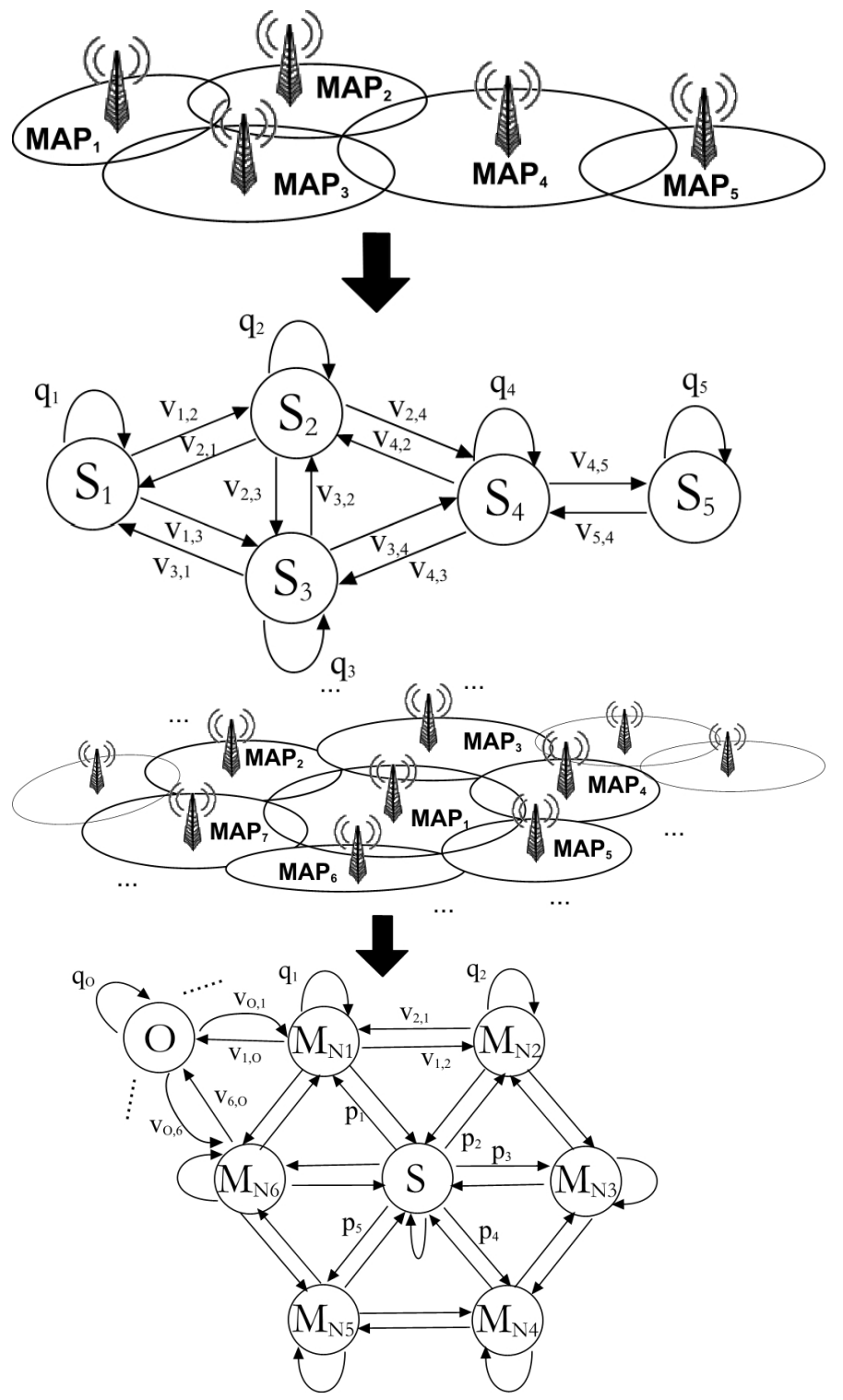

Fig. 2. The creation of models.(a) User-centralized (b) Access pointcentralized/Unstructured/Mn model

the area of the observed MAPs are investigated and their distribution is used to build a model for prediction.

Two guidelines exist:

- In the structured model certain MAPs are grouped together for a predefined reason, which creates a regular structure in the model.

- a MAP or MAPs are simply mapped into a state of Markov model. This method called unstructured model.

Details and examples are presented in the next subchapters.

\subsubsection{Unstructured Markov models (AC/U)}

In the unstructured models a MAP is simply mapped into a Markov-chain state. The results from determined stationary distribution are easy to map back, into the MAPs which makes it simple to use for CAC decisions.

Fig 3 depicts the methodolgy of unstructured model creation. The Markov chain on Fig. 3 shows our previous work [13]. 


\subsubsection{Structured Markov models (AC/S)}

In the structured Markov model groups of MAPs are defined. The grouping can be derived from user behavior, geographical specialty or even network requirement. Fig. 4 and Fig. 5 show examples for structured solutions.

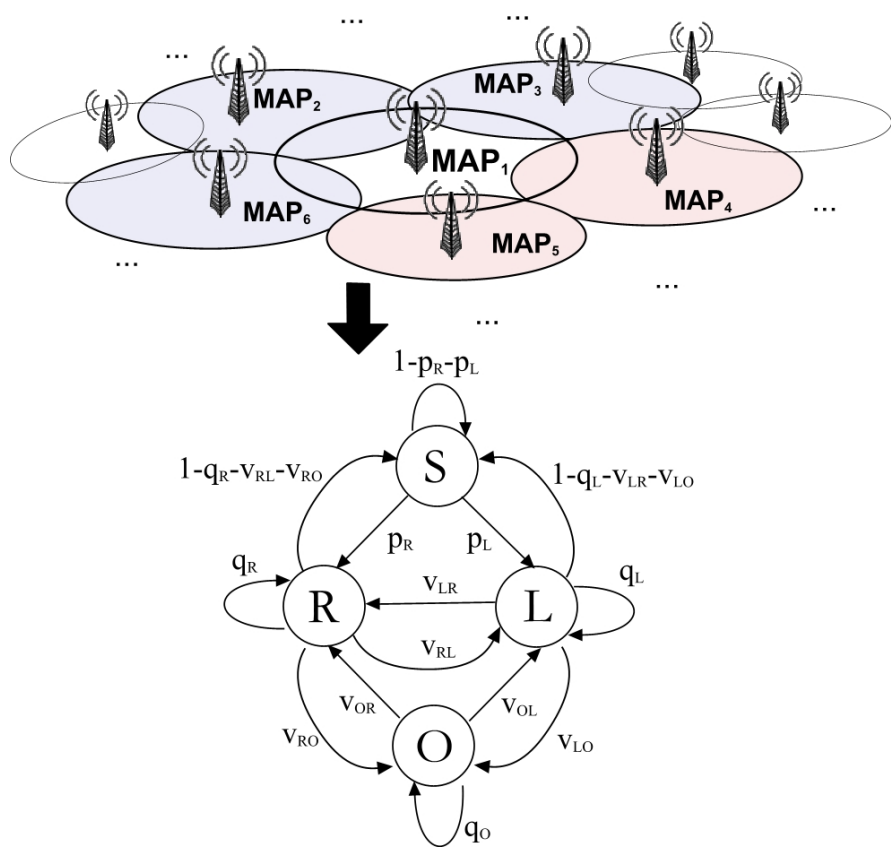

(a)

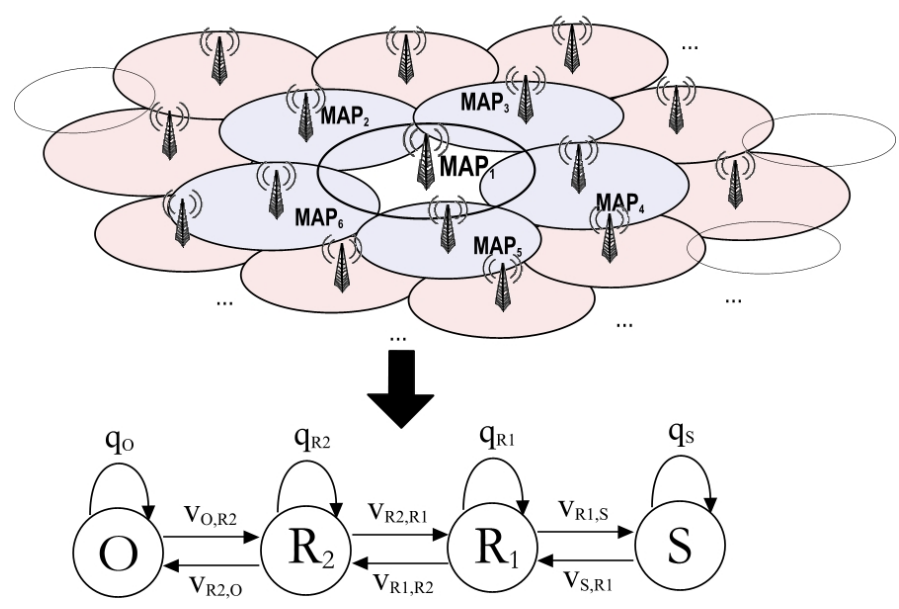

(b)

Fig. 3. The creation of access point-centralized/structured models.(a) M3 model (b) Ring model

The ring model on Fig. 5 is designed for a simple and efficient CAC based in [14]. The M3 model that can be seen on Fig. 4 is also improved as compared to our previous work [13].

\subsection{Attributes}

The example models introduced above are the simplest ones in their class. In this section we determine attributes to the classifying system which describe important parameters of the Markov models. Supported by these attributes more complex, more sophisticated models could be constructed for solving more difficult problems.

At the introduction the examples used present only one at- tribute at a time to keep the simplicity and distinctness. Of course the attributes could be used together in any number and combination.

\subsubsection{Level of the model}

As mentioned in Section 3.2, $\mathrm{B}_{\Pi}$ could be determined from the $\mathbf{P}$ matrix for every status as well. There are two main reasons to handle the statuses differently:

- the users behave differently in certain statuses

- the users in distinct statuses must be modeled in a different way (for example different $\mathrm{CAC}$ is used for the users in voice call, than the users downloading data from the internet)

In these cases the $\mathrm{B}_{\Pi}$ must be calculated for different statuses. This diversity in the model is represented by 'levels' (Fig. 4a). The transition rates between the levels show the intensity status changes in the current MAPs.
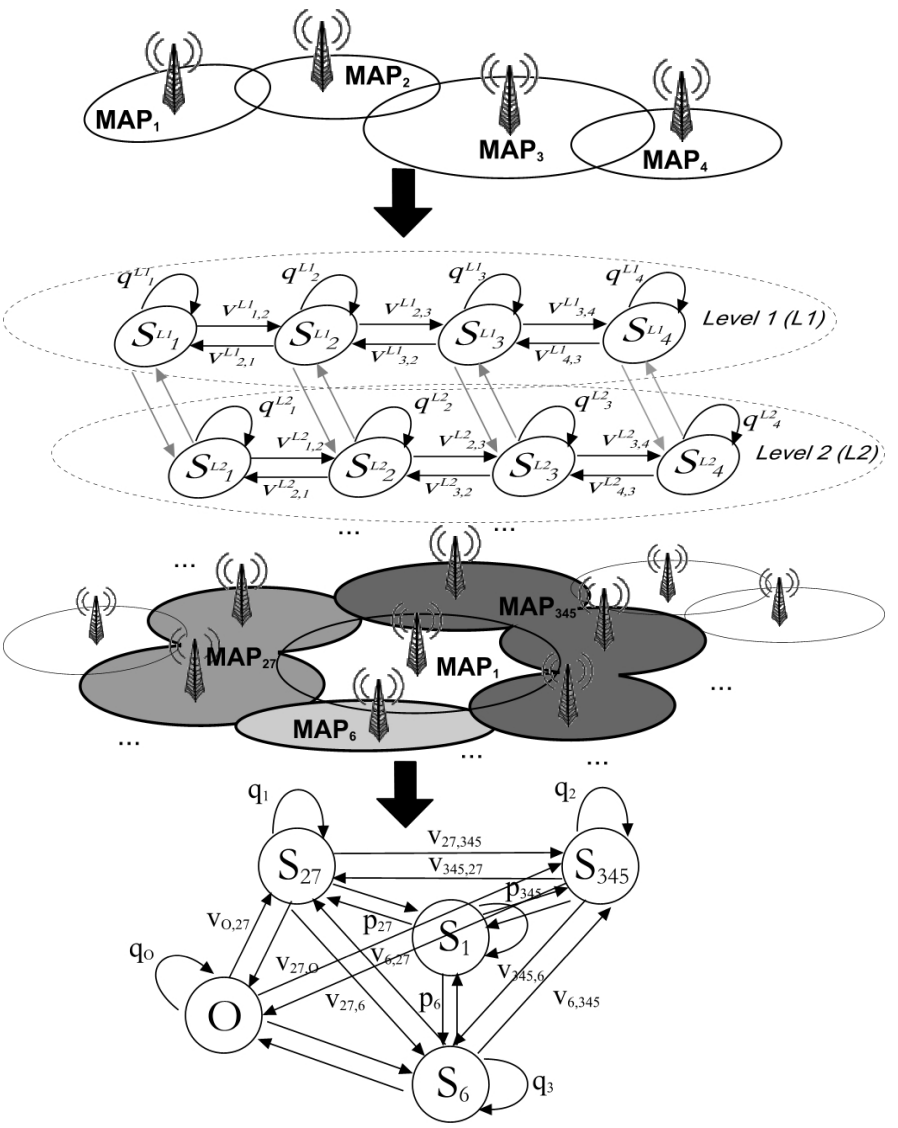

Fig. 4. Attributes in Markov mobility model classifying. (a) Example for meaning of 'level' (b) Example for meaning of resolution

The number of levels in the model is determined with $n_{L}$. A level is denoted with $L$, the levels in the model are marked with $L$ vector, where $L=\left[L_{1}, \ldots, L_{n_{L}}\right]$. A specific $L$ is based on its $B_{\Pi}^{s}$ matrix.

\subsubsection{Resolution of the model}

There is a possibility to merge adjacent MAPs together, if those MAPs are not needed to be handled separately. If outgoing predictions of users in two adjacent MAPs match within a 
certain limit, the two adjacent MAPs could be merged together and handled henceforward as a new major MAP. By this the complexity of the model can be decreased. Fig. ?? shows an example, in which the 6 neighbor MAPs of an access pointcentralized, unstructured model are merged into 3 new major MAPs.

'Grouping' explained in Section 4.3.2 (structured models) is not equal to 'merging' mentioned here. During the 'merging' new, major MAPs are created instead of the initial ones. A 'grouping' organizes the MAPs into a structure.

Every level could have its own resolution. The resolution is denoted with $R, R=G_{1}, \ldots, G_{n_{M}}$, where $G$ is a set of merged MAPs, and $n_{M}$ the number of new MAPs after merging. The $R$ is described a general rate, $n_{m}: n_{M}$. The vector $\mathbf{R}=\left[R_{1}, \ldots, R_{n_{L}}\right]$ contains the resolution rules to every level.

\subsubsection{Order of the model}

The application of the recent user locations has a capital importance in variable, directional user motion. Neglecting the preceding transition series of a user in the MAP results that the estimation works with a significantly higher error rate [13].

Therefore, an $o$-th order could be determined for our Markov models similar like in [4]. In our model, sequence of MAP IDs can be assigned to every MAP not to a user; $I D_{1}, I D_{2}, \ldots, I D_{i}$, $\ldots$, where $I D_{i}$ denotes the identity of the MAP visited by the mobile before it stepped into the current MAP. The last element of the sequence is always the current MAP. The future locations of the mobile in most of the cases are correlated with its movement history. The probability that the user moves to a particular MAP depends on the location of the current cell and a list of cells recently visited. If only the current cell is taken in account, like in previous examples, the order is 1.

For every MAP different $o$-th order could be assigned, which determines the length of the recently visited MAP ID list before the current MAP. Since a MAP could be reached on different paths by the users, therefore more specific MAP ID list could belong to a MAP, and for this reason more Markov state assigned to a MAP, see Fig. 5

Thus $\mathbf{O}=\left[O_{1}, \ldots, O_{n_{c}}\right]$ matrix denotes the order of the model for a level, where oi is the applied sequence length of previous visited MAPs to $\mathrm{MAP}_{i}$. Generally the $n_{c} \times n_{L} O$ matrix $(O=$ $\left[O_{1}, \ldots, O_{n_{L}}\right]$ ) belongs to a Markov model.

The weighted average order for a model is the following:

$$
w_{O}=\frac{\sum_{i=1}^{n_{L}} b^{i} O^{i}}{n_{L}}
$$

\section{Markov Model Constructor Framework}

In this chapter we give guidelines to construct a Markov movement mobility model for a network.

Our theorem is that for every $N\langle S, \underline{\underline{C}}, \underline{\underline{D}}, \underline{\underline{B}}\rangle$ network an optimal $M\langle T, \underline{\underline{L}}, \underline{\underline{R}}, \underline{\underline{O}}\rangle$ Markov model can be determined, where $T$
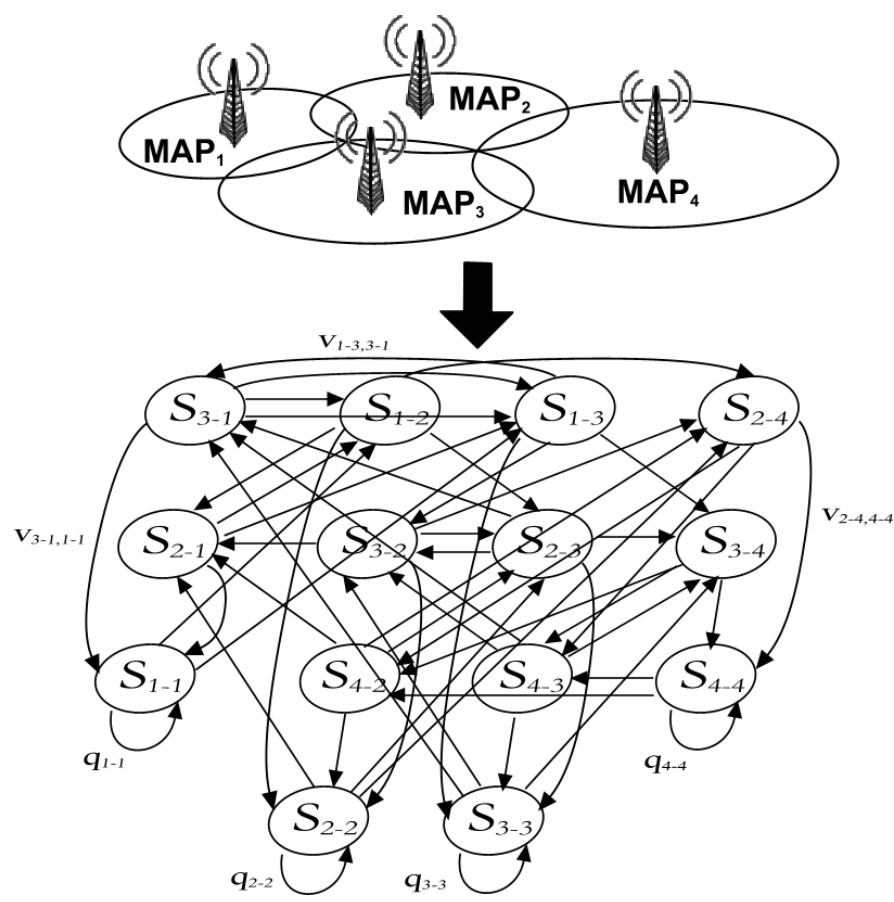

Fig. 5. Example for meaning of 'order'

is the main type of the model, which could be $U C, A C / U$, and $A C / S$.

The next subchapters describe how to determine the attributes and type of the general Markov model in view of the $N$ network.

\subsection{Main type of the model}

The proper main type of the model is determined by the goals and requirements, not by mathematical computation.

Beside the mentioned examples there are some guidelines for selecting the best model type according terms and conditions:

\section{- User-Centralized}

- Modeling from user point of view

- User profile creation

- Fraud detection

\section{- Access Point-Centralized}

- Modeling from cell point of view

- CAC in a MAP

- Movement modeling of a geographical area

\subsection{Determining the level}

A new level should be applied in the model, if the mean difference between transition matrixes for different user statuses is greater than a predefined limit.

Of course if there is a requirement to use levels, than it must applied independently from the calculation.

Let us define $\varepsilon_{d s}$ as limit of mean difference between the $B_{\Pi}$ and transition matrix for different $s$ user statuses $\left(B_{\Pi}^{s}\right)$. The average weighted deviation can be calculated by the following:

$$
w_{d s}^{s}=\frac{b \cdot \operatorname{abs}\left(B_{\Pi}-B_{\Pi}^{s}\right) \cdot 1}{n_{u}^{2}}, s \in S
$$


If $w_{d s}^{s}>\varepsilon_{d s}$ is true for a status $s$, then a new level must be introduced into the model for status $s$. This inequality must be analyzed for all $s \in S$.

The number of levels can be calculated:

$$
\begin{aligned}
n_{L} & =\sum_{\forall s \in S} \operatorname{sign}\left(v_{d s}^{s}+\operatorname{abs}\left(v_{d s}^{s}\right)\right), \\
v_{d s}^{s} & =w_{d s}^{s}-\varepsilon_{d s} .
\end{aligned}
$$

\subsection{Determining order}

The future movement of the users is highly influenced by the path they have taken in the past to reach the investigated point. Leaving this out of consideration would introduce large errors into the mobility model. However it is not always useful to look back into each direction or to look back in equal depth into each direction from every MAP.

The determination of order needs proper precaution. The order exponentially increases the number of states in the model. This can be seen on Fig. 5, where the order is generally 2, for all MAPs $(\mathbf{O}=[2,2,2,2])$.

Main idea is to analyze the importance of each MAP sequence, visited by the users and decide its importance for consideration. The analysis starts with a sequence of length 2 (Length 1 means that only the current MAP is observed) and it is increased one by one. If a sequence of length $i$ belongs to a MAP that is not important, then it will decreased, and $i-1$ order will denoted for the MAP. The importance of $k$ order is decided based on the following basic criteria:

- Take the MAP ID sequences for $k$ length, which differ in the first MAP ID and belong to a current MAP. The difference of the outgoing probabilities of the sequences from current MAP must be investigated. Let us define $\varepsilon_{o p}$ as a limit for this difference. The difference for a $M A P_{i}$ and $k$-order is determined the following way:

$$
\begin{aligned}
& D f[o p]_{i}^{k}= \\
& \frac{\sum_{b \in Q_{i}^{k}} \sum_{a \in Q_{i}^{k}} \frac{\sum_{l \forall N B_{i}}\left|b_{q(a), l}-b_{q(b), l}\right|}{n_{n m}^{i}}}{\left(w_{v n m}^{k}\right)^{2}}
\end{aligned}
$$

where $Q_{i}^{k}$ is the set of existing $k$ length sequences from $M A P_{i}$, $N B_{i}$ is the set of neighbor MAP IDs of $M A P_{i}$ and $q$ denotes a sequence from the set.

The first criterion of importance is: $D f[o p]_{i}^{k}>\varepsilon_{o p}$.

- Take the MAP ID sequences for $m$ length, which differ in the first MAP ID and belong to a current MAP. The fluctuation of the number of users arriving from a certain sequence directions is investigated. Let us define $\varepsilon_{u v}$ as a limit for this variance. The variance of number of incoming users from a sequence into $M A P_{i}$ :

$$
V_{i}^{a}=E\left(\left|E\left(\frac{n_{u}^{i, a}}{n_{u}^{i}}\right)-\left(\frac{n_{u}^{i, a}}{n_{u}^{i}}\right)\right|\right)=\sigma\left(\frac{n_{u}^{i, a}}{n_{u}^{i}}\right)
$$

where $n_{u}^{i, a}$ is the number of users in $M A P_{i}$ arrived from a sequence (path), $n_{u}^{i}$ is the number of users in $M A P_{i}$.

This must be examined for all of the incoming sequences:

$$
D f[u v]_{i}^{k}=\frac{\sum_{a \in Q_{i}^{k}} V_{i}^{q(a)}}{w_{v n m}^{k}}
$$

The second criterion of importance is: $D f[u v]_{i}^{k}>\varepsilon_{u v}$.

This two criteria, $D f[o p]_{i}^{k}>\varepsilon_{o p}$ and $D f[u v]_{i}^{k}>\varepsilon_{u v}$ must be applied for all MAP in order to determine $\mathbf{O}$.

\subsection{Determining resolution}

We explained earlier that if outgoing predictions of users in two adjacent MAPs matches within a certain limit, then the MAPs could be merged together to create a new, major MAP.

In our previous work we already published an algorithm for resolution determination. Because of the limitations of this paper the details can be seen in [13].

\subsection{Complexity}

The complexity of the model could be denoted with the number of states. Following the determining of attributes the number of states is:

$$
n_{\text {States }}=\sum_{\forall l \in L} \frac{n_{m}^{l}}{n_{M}^{l}} \cdot w_{v n m}^{l} w_{o}^{l}
$$

\section{Numerical results}

In this chapter we compare the accuracy of the proposed MMCF to other models found in the literature. The estimation procedure was validated by a simulation environment of a cell cluster shown on Fig. 6

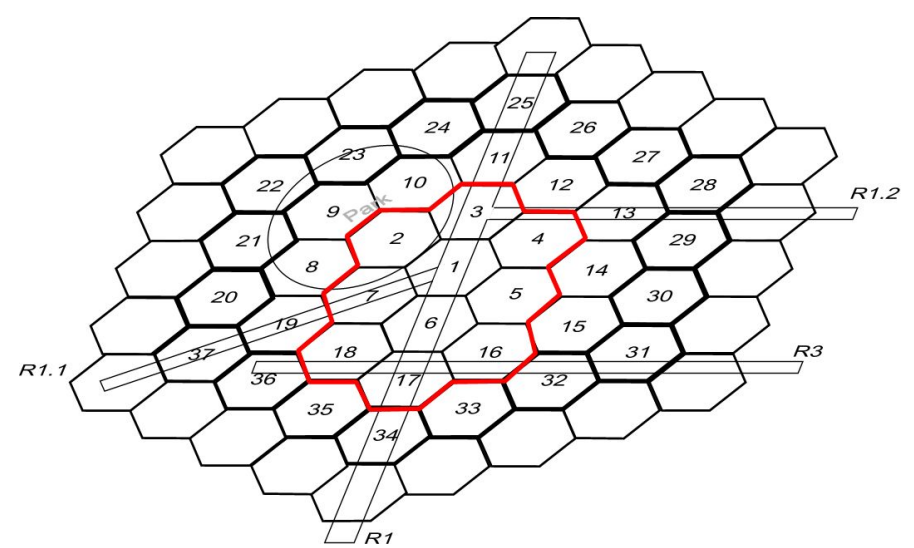

Fig. 6. Cell-cluster and the examined area in the simulation environment.

The simulation was written in the open source OMNet++ using $\mathrm{C}++$ language. The simulation environment consisted of a cluster with 61 named cells and it also included geographical data that is interpreted as streets and a park on the cluster area. The drift of the movement is heading to the streets from neutral areas.

The simulation used 610 mobile terminals (10 for each cell), in the initial state uniformly distributed in the cluster. The average motion velocity of the users is parametrized with a simple 
phase-type $(\mathrm{PH})$ cell dwell time simulator (reciprocal of exponentially distributed values). In the simulation time mobile terminals appear and disappear, in order to simulate the active and inactive states.

The simulation consists of two parts. The trace simulation is the series of cell-transitions that the mobiles have initiated. It produces a time-trace that contains the actual location data for each mobile terminal in the network (reference interval). We have used this trace simulation as if it was a provider's real network trace.

The second part is the estimation procedure that uses the past and the current reference simulation results to estimate future number of users in each cell. The estimation error is interpreted as the measure of accuracy of each mobility model in this paper.

The prediction starts 100 timeslots after the reference simulation initiation. During the warm-up process the reference simulation produces enough sample data for the correct estimation, which uses the previous reference results as an input to estimate the future user distribution. Each user-transition in the 100-timeslot reference period is used to derive transition probabilities, motion speed and patterns in the simulation cell-space. These patterns serve as an input for the simulation threads of each mobility model. The models have the same input throughout the simulation process so that the results are comparable.

A widely used modified Random Walk estimation, M3 [14] and M7 [13] models were used in the simulation as references. The examined area contains the cells in the circle (cell 1-7, cell 16-18) Fig. 6

The input parameters of MMCF for this simulation environment: $\mathrm{S}=$ handover during voice call, $\varepsilon_{C}=5, \varepsilon_{u v}=0.4$, $\varepsilon_{o p}=0.2$,

We examined only the handover event, so the $\mathrm{D}$ matrix is empty and because of the limits of this paper the C and BQ matrices are not presented. Structured, access point centralized model with one level was chosen. The result of the algorithms:

$\underline{\underline{O}}=[2,1,1,1,2,1,1,2,1]-$ which means for MAPs $1,6,17$ the order is 2 , for the others it is 1 ,

$\underline{\underline{R}}=[1,2,3,4,5,6,7,16,17,18]-$ which means that MAPs 4 and 5 are merged together and the Markov-chain is the following (for clear interpretation not all of the edges depicted):

The following plots (Fig. 8) show the avarage error of the estimations in every $t$ timeslot. Random Walk model (RW line) peformed worst, it cannot follow the patterns in user fluctuation as it was expected. The M3 (M3 line) and M7 (M7 line) models work with significantly lower error rate, but in $t=5$, $t=20$ and $t=32$ timeslots the average error rate increased suddenly. This is caused by the change of distribution of the directional moving users (suddenly increased the number of active mobile users), what the simple Markov models cannot follow. The MMCF generated Markovian approach (MMCF line) holds the avarage error rate, it followed the changes in user motion appropriately, it is able to learn the directional motion patterns and the fluctuation of user distribution, which proves the strength of

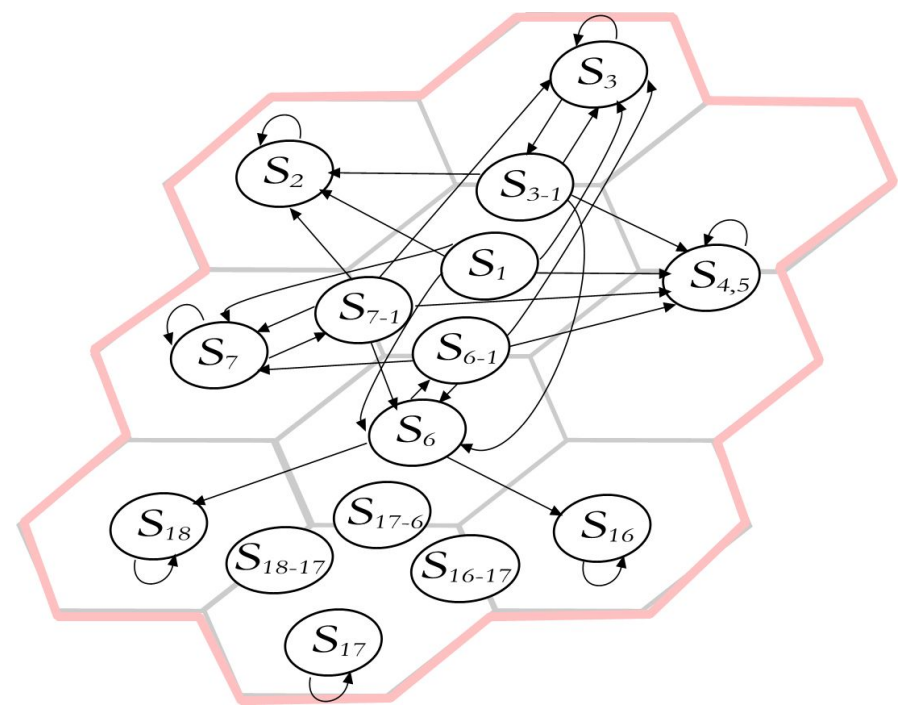

Fig. 7. Markov-chain of the generated model by MMCF.

the Markov Model Creator Framework.

\section{Conclusion}

In this paper we grabbed some significant parameters of mobility and proposed a method to model the mobile node and the network independently of the technology used. We proposed a simple classifying for Markov mobility models, and we have shown examples for the most important types. We showed the attributes of a general Markov model, and we prepared processes for definition. Obviously these algorithms could be further refined. Using the framework it is not necessary to create a new Markov model, only the description of the network, parameters and the requirement of the accuracy must be given and a Markov movement model is generated with minimal number of states. The network operator may use this Markov model to make predictions on the future distribution and location of users among radio cells to justify CAC or other QoS decisions or support self-configuring system in $4 \mathrm{G}$ mobile networks.

\section{References}

1 Yu F, Leung V C M, Mobility-based Predictive Call Admission Control and Bandwidth Reservation in Wireless Cellular Networks, Elsevier Computer Networks 38 (Apr. 2002), no. 5, 577-589, DOI 10.1016/S13891286(01)00269-9.

2 Lin $\mathbf{Y} \mathbf{B}$, Chen $\mathbf{N}$, Rao $\mathbf{H}$, Potential Fraudulent Usage in Mobile Telecommunications Networks $\mathbf{1}$ (2002), no. 2, 123-131, DOI 10.1109/TMC.2002.1038348.

3 Debar H, Dacier M, Wespi A, A Revised Taxonomy for Intrusion-Detection Systems, Annales des Telecommunications 55 (2000), 361-378.

4 Sun B, F. Yu F, Wu K, Leung VCM, Mobility-Based Anomaly. Detection in Cellular Mobile Networks, ACM WiSe'04, posted on 2004, 61-69, DOI 10.1145/1023646.1023658, (to appear in print).

5 Dottling M, Viering I, Challenges in Mobile Network Operation: Towards Self-Optimizing Networks, Proc. IEEE Int'1. Conf. Acoustics, Speech, and Sig. Processing, posted on Apr. 2009, 3609-12, DOI 10.1109/ICASSP.2009.4960407, (to appear in print).

6 Haas Z, A new routing protocol for reconfigurable wireless networks, In Proceedings of the IEEE International Conference on Universal Personal Com- 


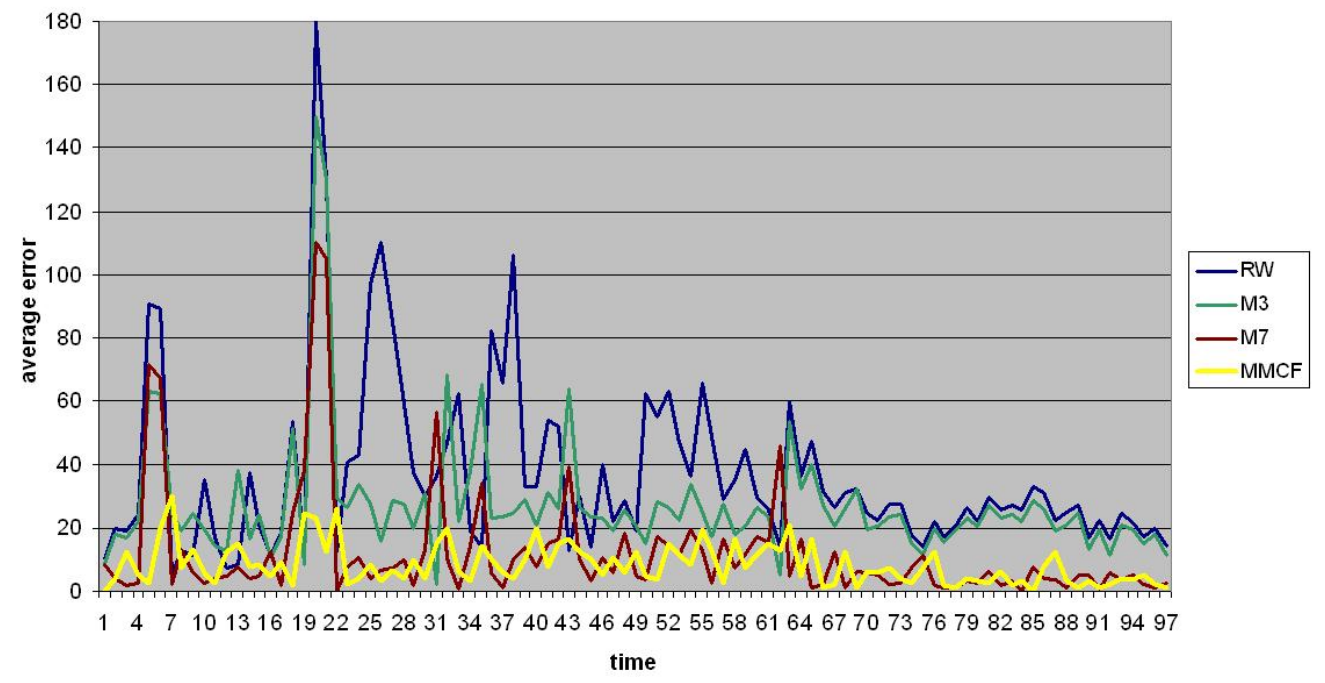

Fig. 8. The average error of the different models.

munications (ICUPC), posted on 1997, DOI 10.1109/ICUPC.1997.627227, (to appear in print)

7 Hong X, Kwon T, Gerla M, Gu D, Pei G, A Mobility Framework for Ad Hoc Wireless Networks, In proceedings of ACM 2nd International Conference on Mobile Data Management, (MDM 2001), posted on 2001, January, DOI 10.1007/3-540-44498-X_15, (to appear in print)

8 Sun B, Yu F, Wu K, Xiao Y, Leung V C M, Enhancing Security using Mobility-Based Anomaly Detection in Cellular Mobile Networks, IEEE Transactions on vehicular technology 1, DOI 10.1109/TVT.2006.874579, (to appear in print).

9 Deshpande M, Karypis G, Selective Markov models for predicting web page accesses, ACM Transactions on Internet Technology (TOIT) 4 (May 2004 ), no. 2, 163-184, DOI 10.1145/990301.990304.

10 Lassabe F, Charlet D, Canalda Ph, Chatonnay P, Spies F, Predictive Mobility Models based on Kth Markov Models, In IEEE Int. Conf. on Pervasive Services 2006 (ICPS'06), posted on June 2006, 303-306, DOI 10.1109/PERSER.2006.1652248, (to appear in print).

11 Fülöp P, Kovács B, Imre S, Mobility Management Algorithms for the Client-driven Mobility Frame System - Mobility from a Brand new point of view, Mobile Information Systems (2009), 313-337.

12 Fülöp P, Kovács B, Imre S, Enhanced Mobility Management Modelling Framework, Proc. 6th Computer Information Systems and Industrial Management Applications (CISIM 2007), posted on June 28-30 2007, 53-58, DOI 10.1109/CISIM.2007.26, (to appear in print).

13 Fülöp P, Szálka T, Szabó S, Imre S, Accurate Mobility Modelling and Location Prediction based on Pattern Analysis of Handover Series in Mobile Networks, Mobile Information Systems, posted on 2009, 1-35, DOI 10.1145/1497185.1497232, (to appear in print).

14 Szabó S, The effects of user mobility on the performance of wireless networks (2010). Ph.D dissertation.

15 Shiang-Chun Lion, Queh-Min Huang, Trajectory Predictions in Mobile Networks, International Journal of Information Technology 11 (2005), no. 11.

16 Wencho Ma, Yuguang Fang, Phone Lin, Mobility Management Strategy Based on User Mobility Patterns in Wireless Networks, IEEE Transactions on Vehicular Technology 56 (January 2007), no. 1, 322-330, DOI 10.1109/TVT.2006.883743. 